\title{
Deep Residual Learning for Analyzing Customer Satisfaction using Video Surveillance
}

\author{
Nehemia Sugianto \\ Southern Cross University \\ Southern Cross Dr, QLD 4225 \\ nehemia.sugianto@scu.edu.au
}

\author{
Dian Tjondronegoro \\ Southern Cross University \\ Southern Cross Dr, QLD 4225 \\ dian.tjondronegoro@scu.edu.au
}

\author{
Beau Tydd \\ Queensland Airports Limited \\ Eastern Avenue, QLD 4225 \\ btydd@qldairports.com.au
}

\begin{abstract}
Measuring customer satisfaction based on facial expressions from video surveillance can potentially support real-time analysis. We propose the use of deep residual network (ResNet), which has been a widely used for many image recognition tasks, but not in the context of recognizing facial expressions in video surveillance. A key challenge in collecting video surveillance data in an airport context is to achieve a balanced distribution of all emotions, as most of passengers' faces are either neutral or happy. To solve this issue, there is no existing work that has established the feasibility of using datasets from different domains to train the model. This paper is the first in investigating the benefits of using residual training approach and adopt a pre-trained network from similar tasks to reduce training time. Based on comprehensive experiments, which compare domain-specific, crossdomain and mixed domain training and testing approaches, we confirm the value of augmenting datasets from different domains (CK+, JAFFE, AffectNet) for the surveillance domain.
\end{abstract}

\section{Introduction}

Customer satisfaction can be measured using manual methods such as survey, interview and focus group, which can be obtained at the point of service to capture prompt feedback, or at specific intervals to capture the overall measurement and feedback. These manual measurements are limited to interviewers' subjective performance and record-keeping skill, and the restricted scope of duration and frequency of data collection may not capture the customers' accurate feedbacks.

Facial expression plays an important role in human communication due to its ability to convey a reaction to the surrounding environment. In the context of customer satisfaction, a lower perceived service quality is often accompanied by negative customer emotions, such as angry and sad emotions [1]. $70-95 \%$ of dissatisfied customers do not complain verbally [2] and a facial expression constitutes $55 \%$ of the intended emotion in a spoken message [3]. In addition to these advantages, facial expressions can be readily analyzed from video surveillance, which is less interfering than attaching biometric sensors to a person for affective detection. However, automatic facial expression recognition (FER) on video surveillance is still at an infancy stage. Collecting and labelling a large-scale spontaneous FER dataset has only been recently achieved for general purposes, which has not included surveillance application domains [4].

Deep learning has been shown to be effective for FER tasks, capable of automatically classifying facial expressions without the need to manually engineer features [5]. However, there are two main challenges in building a robust deep-learning based FER model for video surveillance: 1) overcoming domain-specificity issue $[6,7]$ which means that the model must be trained on video surveillance dataset; and 2) the model will have a good performance if it is trained on a large dataset and balanced distribution of all emotions [6]. Existing datasets in video surveillance are only labelled for face recognition tasks. Hence, we have constructed a new dataset for FER in video surveillance specifically for customer experience measurement purpose, which focused on labelling happy, angry, and neutral classes. We have collected 16,575 neutral samples and 3,307 happy samples but only 27 angry samples, out of 20,474 faces from 2-day video footages. To overcome the risk of unbalanced sample distribution per class, we explore the feasibility of training the deep learning model by augmenting existing FER datasets from different domains.

To train a deep learning model using a limited dataset, one approach is to adopt existing pre-trained model and fine-tune it to work on the target problem domain, known as knowledge transfer. This paper proposes a deep residual learning model inspired by ResNet [8], which has been trained on a large dataset (ImageNet) to classify objects based on visual characteristics. As the visual features used for object and facial expression are quite similar at the low-to-mid level (e.g. lines, edges), then we expect to benefit from reusing most of the knowledge formed in the first layers of the network. The key contribution is to 
investigate the feasibility of domain-specific learning on the last few layers of the network, to speed up the learning process and reuse the gained knowledge from similar task. The experiment results confirm that the models trained by $C K+[9,10], J A F F E$ [11] and AffectNet [4] datasets can be used for FER in video surveillance domain without the need of retraining all of the deep neural network's layers.

\section{Related Works}

Deep learning model, specifically deep convolutional neural network $(\mathrm{CNN})$, has become the state-of-the-art technique for FER tasks, thanks to its capability to automatically extract subtle features that can be missed by domain experts. Deep-learning based FER has been proposed to work with either individual images $[5,12-16]$ or sequence of images to exploit the spatio-temporal information between images [17], both of which have been shown to outperform conventional (manually engineered) models. However, the absence of a large domain-specific dataset is still the main challenge in training a deeplearning based FER models for video surveillance context, as most of existing datasets are captured in a lab-controlled environment. Most of recent works of deep learning in video surveillance focus on face recognition [18-21].

Previous studies, such as [13] and [22], confirmed that knowledge transfer and augmenting data from different domain datasets approaches can be used to build deeplearning models with a small dataset. It means that we can adopt an existing pre-trained model from similar tasks and fine-tune it to perform on a target problem domain.

One of the most well-known approaches to improving deep learning model performance is by adding more layers. However, once the model becomes deeper, it also requires longer training time as the number of parameters trained is increasing as well. This approach may actually lead to worsening the accuracy level. When the model depth increases, the accuracy gets saturated and then degrades rapidly. Some neurons in some cases can "die" during training and become ineffective, which is known as the effect of vanishing gradient problem. Inspired by VGG [23], ResNet addresses this degradation problem by proposing a residual connection in the convolutional layers, known as the shortcut connection. It enables us to carry important information from the previous layers to the next layers and reduce information loss and surprisingly fasten the training time. The model has fewer parameters compared to VGG model ( 8 times smaller in depth).

\section{Methodology}

To analyze customer satisfaction, our system has three main steps: 1) face detection to extract face region(s) found in each video frame; 2) FER to recognize facial expression (neutral, happy or angry) from each face region, and 3) face verification to keep track of a customer's journey across time and multiple camera locations. A summary report is generated using the outputs showing the journey experience of each passenger over time. This paper will focus on the FER as a key component of the system.

FER is more sensitive compared to object recognition as facial expressions are located mostly on eyes and mouth and formed a small fraction of a frontal face image [24]. Deeper model is required to be able to recognize the subtle features. Among its variants, ResNet50, ResNet101 and ResNet152 are more accurate than ResNet18 and ResNet34 [8]. ResNet50 has fewer number of parameters $(0.85 \mathrm{M})$ compared to other deeper models $(>1.7 \mathrm{M})$, but still has high performance on object classification task ( $0.96 \%$ in difference), thus the model is faster to converge. For practical applications, memory constraint and execution time are important considerations for selecting the model.

The proposed model architecture is based on ResNet50 as depicted in Figure 1. The network has five convolutional layers, in which the last four layers are used differently as the building blocks. Shortcut connection is adopted in each building block to prevent degradation problem by passing important information from previous layers to the next layers. Down sampling is performed in the last three building blocks to prevent overfitting. The network ends with a global average pool and N-output fully-connected layer containing a SoftMax activation.

Transferring knowledge from pre-trained network: We adopt the ResNet50, which has been trained using ImageNet dataset $(>1.2 \mathrm{M}$ object images) to classify objects into 1,000 different object classes. We have experimentally tested that this approach is faster than adopting the original (untrained) ResNet50 for training towards FER task. To transfer the gained knowledge, we need to retrain the few last layers of the pre-trained network. Based on the TensorFlow implementation, which is composed of 176 layers, we proposed four different models (last-5, last-10, last-50, and last-75), in order to determine how far we need to fine tune the last $N$-layers of the network, except the last layer (the fully connected layer). Final layers are chosen as object and facial expression recognition have different visual features at high-level. Finally, the last layer is modified to 3 neurons (neutral, happy and angry).

Augmenting existing datasets from different domains: due to the limited amount of labelled video surveillance data, we use three different strategies to train the model using augmented training datasets: 1) cross domain, 2) mixed domain, and 3) domain specific. The next sections will evaluate the most effective strategy, while investigating the impact of mixing data. 


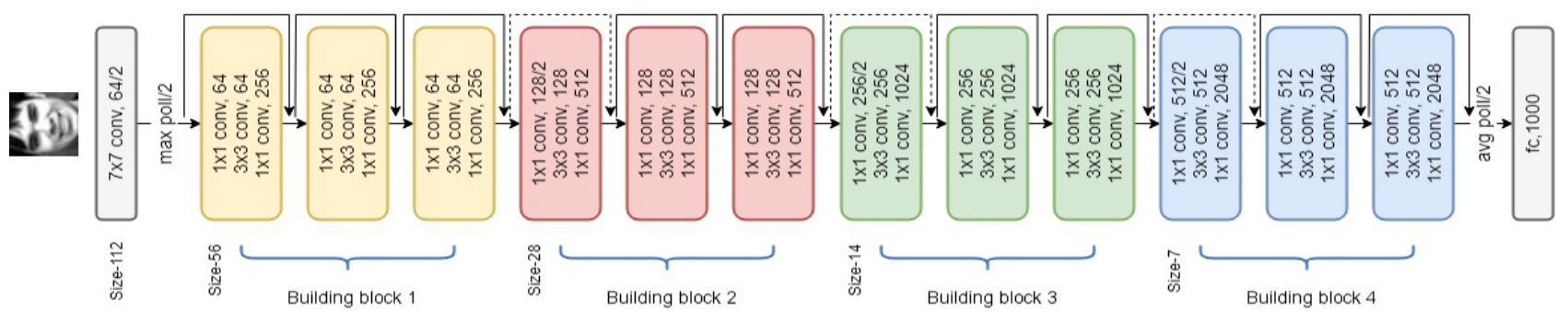

Figure 1. ResNet-50 architecture

\section{Experimental Setup}

Our experiments have three main objectives: 1) to investigate if residual deep-learning based FER model can work on video surveillance context, 2) to evaluate which model has the most optimal effect of knowledge transfer from ImageNet, and 3) to investigate the effectiveness of augmenting existing datasets from different domains to overcome surveillance data limitations.

\subsection{Datasets}

The experiments use four datasets: 1) $\mathrm{CK}+$, 2) JAFFE, 3) AffectNet, which have been used as FER datasets for different applications, and 4) a new video surveillance dataset specifically created for the target domain application. The first two datasets represent posed and non-spontaneous expression (lab-controlled), while the rest are non-posed and spontaneous expression (in-the-wild). Sample images of each class from the different datasets can be found in Table 1. Video surveillance images are inverted to obscure the faces and anonymize the subjects.

\begin{tabular}{|c|c|c|c|c|}
\hline Dataset & \multicolumn{3}{|c|}{ Image samples } \\
\hline OK+ \\
Original size: \\
$204-349$ pixels
\end{tabular}

Table 1. Sample images of each dataset after pre-processing

$\mathbf{C K}+$ is one of the most used dataset for FER, composed of 593 image sequences from 123 subjects and is classified into seven basic emotions. It does not have any neutral emotion images and mostly are grayscale images.
JAFFE contains 213 grayscale images of 7 posed facial expression (6 basic facial expression and neutral) that are posed by 10 Japanese female models. Each image has been rated on 6 emotion adjectives by 60 Japanese subjects.

AffectNet contains $\sim 1 \mathrm{M}$ spontaneous and color facial images collected from the Internet by querying three major search engines using 1,250 emotions related keywords in six different languages. About half of the retrieved images $(\sim 420 \mathrm{~K})$ are manually annotated for the presence of seven discrete facial expression (categorical model) and the intensity of valence and arousal (dimensional model) by human experts. The rest of the images $(\sim 550 \mathrm{~K})$ are automatically annotated using ResNet neural network trained using all the manually annotated training samples.

Video surveillance is newly collected by capturing live video surveillance at a local airport. We choose to extract footages from cameras located at parking gate, security and check-in areas, as they show the clearest facial images of the passengers. Viola-Jones's face detection is used to extract the faces regions. To save data collection time and cost, three automatic annotators (Azure[25], Face++[26] and Emovu[27]) are used to label the faces into 7 basic and neutral emotions. Samples are selected from the highest value with a threshold of 0.4 and when all annotators agree on a single emotion type.

Image samples selected for experiments: the experiments select samples from three classes: neutral, happy and angry. These emotions represent the most occurring positive and negative emotions from customers at an airport. To ensure data quality and reduce noises, we only use facial images with dimension of 55 to 225 pixels from the video surveillance dataset. From the AffectNet dataset, we only use manually annotated images. Hence, the sample class distributions for each dataset used in our experiments are shown in Table 2.

\begin{tabular}{|c|c|c|c|c|}
\hline Dataset & \#Image & Neutral & Happy & Angry \\
\hline CK+ & 114 & $0(0 \%)$ & $69(61 \%)$ & $45(39 \%)$ \\
\hline JAFFE & 91 & $30(33 \%)$ & $31(34 \%)$ & $30(33 \%)$ \\
\hline AffectNet & 1,800 & $600(34 \%)$ & $600(33 \%)$ & $600(33 \%)$ \\
\hline $\begin{array}{c}\text { Video } \\
\text { surveillance }\end{array}$ & 327 & $150(46 \%)$ & $150(46 \%)$ & $27(8 \%)$ \\
\hline
\end{tabular}

Table 2. Comparison of four datasets (\#sample vs percentage) 


\subsection{Pre-processing}

All images are converted into grayscale images and cropped using Viola-Jones' face detection algorithm [28] to get the smallest rectangular area containing the face region and then resized to $224 \times 224$ pixels. We also perform data augmentation on $\mathrm{CK}+$, JAFFE and airport video surveillance datasets to increase the number of images of training and validation sub datasets by performing horizontal flip.

\subsection{Training and testing}

The proposed models are implemented using Tensorflow on a NVIDIA Tesla K80 GPU and takes roughly 24 hours per experiment as number of trained layers affects the number of hours to train the models. The more layers are trained, the longer time to train the model as it performs more complex computation and requires more memory resource.

The accuracy of the proposed FER models is evaluated using a K-fold cross validation technique on CK+, JAFFE, AffectNet datasets, with $\mathrm{K}=10$. For video surveillance dataset, the model is evaluated using 1-fold cross validation. Our models are trained using same hyper parameters and SGD (Stochastic Gradient Descent) optimizer. The decay is $5.10^{-4}$ and momentum is 0.9 using Nesterov momentum. We experimentally determine that $1.10^{-2}$ is the best learning rate to train the models. The learning rate can be reduced to $1.10^{-3}$ when the validation accuracy is on a plateauing stage with factor of 0.5 and patience is 10. Each dataset is divided into $90 \%$ for training and validation, and $10 \%$ for testing purposes, with no overlap. Images for each output class are mutually exclusive as there is no overlapping images in more than one class. Table 3 summarizes how we conduct three experiments to investigate the benefits of augmenting existing datasets from different domains.

\begin{tabular}{|c|ll|}
\hline Experiment & \multicolumn{1}{|c|}{ Strategy } \\
\hline Experiment 1: & $\bullet$ & Train using video surveillance \\
Domain-specific & $\bullet$ & Test on video surveillance \\
\hline Experiment 2: & $\bullet$ & Train using CK+, JAFFE or AffectNet only \\
Cross domain & $\bullet$ & Test on video surveillance \\
\hline Experiment 3: & $\bullet \begin{array}{l}\text { Train using CK+, JAFFE, or AffectNet } \\
\text { first, and then video surveillance }\end{array}$ \\
\hline Mixed domain & $\bullet \quad$ Test on video surveillance \\
\hline
\end{tabular}

Table 3. Proposed experiment strategies to train/test the model

\section{Experimental Results}

Based on experiment 1, found that training a deeplearning based FER models using only video surveillance yields a very poor performance. As shown in Table 4, the model can only detect neutral emotions, and most of happy and negative images are incorrectly labelled as neutral.
This is most likely due to not enough angry images and the challenge in using non-laboratory data that is collected across different cameras, with varying resolution and face angles, thereby increasing the likelihood of training data noises.

\begin{tabular}{|c|c|c|c|c|c|c|c|}
\hline & \multicolumn{4}{|c|}{ Actual emotions } & \multicolumn{3}{|c|}{ Measurement } \\
\hline \multirow{4}{*}{ 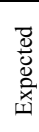 } & $\%$ & $\mathrm{NE}$ & HA & $\mathrm{AG}$ & Prec. & Recall & F1 \\
\hline & $\mathrm{NE}$ & 93.4 & 6.6 & 0 & 0.5 & 0.9 & \multirow{3}{*}{0.48} \\
\hline & HA & 93.4 & 6.6 & 0 & 0.5 & 0.1 & \\
\hline & $\mathrm{AG}$ & 100 & 0 & $\mathbf{0}$ & NA & 0 & \\
\hline
\end{tabular}

\subsection{Performance of knowledge transfer}

Table 5 depicts that the Last- 75 models always achieve the best validation accuracy: $91.41 \%, 85.34 \%$, and $83.10 \%$ on CK+, JAFFE, and AffectNet datasets respectively. This confirms that pre-trained (ImageNet) models can perform better for a new task (FER) when more of the last layers are retrained.

As shown in Figure 2, the Last-75 model can be trained quite efficiently, as it converged after 50-100 epochs, out of the 200 epochs (i.e. 6,000 iterations on mini-batches of size 12 samples). However, we have not experimented training higher than Last-75 layers due to resources constraints. It is expected that we would need to eventually identify the most optimum balance between number of trained layers and the time required to achieve highest possible accuracy.

\begin{tabular}{|l|l|c|}
\hline Dataset & $\begin{array}{l}\text { Number of trained } \\
\text { layers }\end{array}$ & $\begin{array}{c}\text { Average Validation } \\
\text { Accuracy (\%) }\end{array}$ \\
\hline \multirow{5}{*}{ CK+ } & Last-5 & 71.74 \\
\cline { 2 - 3 } & Last-10 & 73.60 \\
\cline { 2 - 3 } & Last-50 & 85.28 \\
\cline { 2 - 3 } & Last-75 & $\mathbf{9 1 . 4 1}$ \\
\hline JAFFE & Last-5 & 70.18 \\
\cline { 2 - 3 } & Last-10 & 72.28 \\
\cline { 2 - 3 } & Last-50 & 82.89 \\
\cline { 2 - 3 } & Last-75 & $\mathbf{8 5 . 3 4}$ \\
\hline \multirow{5}{*}{ AffectNet } & Last-5 & 63.40 \\
\cline { 2 - 3 } & Last-10 & 71.23 \\
\cline { 2 - 3 } & Last-50 & 79.56 \\
\cline { 2 - 3 } & Last-75 & $\mathbf{8 3 . 1 0}$ \\
\hline
\end{tabular}

Table 5. Average validation accuracy (\%). Grey rows denote the best model from each dataset

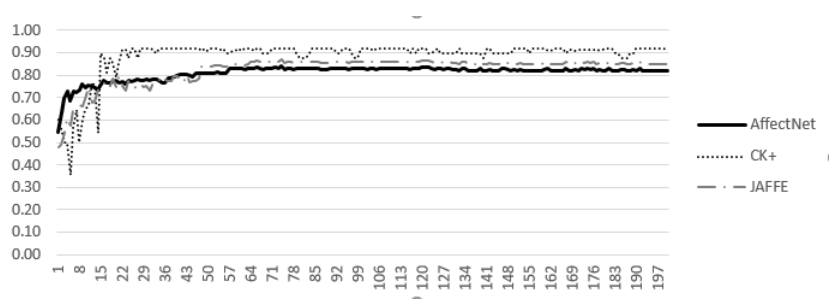

Figure 2. Accuracy of the best model (last-75 model) trained on AffectNet: $\mathrm{x}$-axis indicates \#epoch 


\subsection{Performance of Augmenting Datasets}

To fully investigate the performance of the Last-75 model, the confusion matrix and F1-score for each Experiment 2 and 3 are presented in Table 6 and 7, whereby NE, HA, AG denote neutral, happy and angry emotions respectively.

In-the-wild FER dataset is better suited for video surveillance context: As shown in Table 6, despite the AffectNet trained model has the lowest validation accuracy (83.10\%, compared to $91.41 \%$ and $85.34 \%$ for $\mathrm{CK}+$ and JAFFE trained models respectively, as shown in Table 4), the model has the best F1-score (0.64) when tested on video surveillance dataset, compared to $\mathrm{CK}+$ and JAFFE trained models that achieved 0.20 and 0.38 respectively. This is most likely due AffectNet dataset has a higher similarity with video surveillance dataset, as both datasets represent facial expression in the wild environment.

Trained with $\mathrm{CK}+$, tested on video surveillance

\begin{tabular}{|c|c|c|c|c|c|c|c|}
\hline & \multicolumn{4}{|c|}{ Actual emotions } & \multicolumn{3}{|c|}{ Measurement } \\
\hline \multirow{4}{*}{ 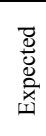 } & $\%$ & $\mathrm{NE}$ & HA & $\mathrm{AG}$ & Prec. & Recall & F1 \\
\hline & $\mathrm{NE}$ & $\mathbf{0}$ & 40 & 60 & 0 & 0 & \multirow{3}{*}{0.20} \\
\hline & HA & 46.6 & 40 & 13.4 & 0.46 & 0.4 & \\
\hline & $\mathrm{AG}$ & 34 & 33 & 33 & 0.08 & 0.33 & \\
\hline
\end{tabular}

Trained with JAFFE, tested on video surveillance

\begin{tabular}{|c|c|c|c|c|c|c|c|}
\hline \multirow{4}{*}{ 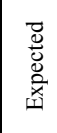 } & $\%$ & NE & HA & $\mathrm{AG}$ & Prec. & Recall & F1 \\
\hline & $\mathrm{NE}$ & 46.7 & 53.3 & 0 & 0.44 & 0.47 & \multirow{3}{*}{0.38} \\
\hline & HA & 53.6 & 33 & 13.4 & 0.36 & 0.33 & \\
\hline & $\mathrm{AG}$ & 33 & 34 & 33 & 0.33 & 0.33 & \\
\hline
\end{tabular}

Trained with AffectNet, tested on video surveillance

\begin{tabular}{|c|c|c|c|c|c|c|c|}
\hline \multirow{4}{*}{ 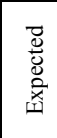 } & $\%$ & $\mathrm{NE}$ & HA & $\mathrm{AG}$ & Prec. & Recall & F1 \\
\hline & 0 & 67.3 & 13.3 & 19.4 & 0.67 & 0.67 & \multirow{3}{*}{0.64} \\
\hline & HA & 26.7 & 66.6 & $\begin{array}{l}6.7 \\
\end{array}$ & 0.83 & 0.67 & \\
\hline & $\mathrm{AG}$ & 34 & 0 & 66 & 0.33 & 0.67 & \\
\hline
\end{tabular}

Table 6. Confusion matrix (\%) of the best model (last-75 model) when tested on video surveillance dataset (Exp. 2)

Cross domain is the best approach in augmenting datasets: As shown in Table 6, the models trained using different domains (specifically AffectNet) have higher F1scores (0.64) compared to when the model is only trained on the target domain (i.e. 0.48 as per Table 4). However, as shown in Table 7, the models become weaker when they are also trained using video surveillance dataset after being trained using a cross-domain datasets. The F1-scores decrease by $0.1,0.19$ and 0.27 respectively. We argue that the varying quality of video resolution and the lack of angry emotion samples in the video surveillance dataset are the main issues that have affected the models' performance. Therefore, using similar datasets with similar characteristics to the target domain could yield a better performance when the dataset has a better image resolution.
Trained with $\mathrm{CK}+$ then video surveillance, tested on video surveillance

\begin{tabular}{|c|c|c|c|c|c|c|c|}
\hline & \multicolumn{4}{|c|}{ Actual emotions } & \multicolumn{3}{|c|}{ Measurement } \\
\hline \multirow{4}{*}{ 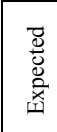 } & $\%$ & $\mathrm{NE}$ & HA & $\mathrm{AG}$ & Prec. & Recall & F1 \\
\hline & $\mathrm{NE}$ & $\mathbf{0}$ & 53.3 & 46.7 & 0 & 0 & \multirow{3}{*}{0.1} \\
\hline & HA & 60 & 26.6 & 13.4 & 0.31 & 0.7 & \\
\hline & $\mathrm{AG}$ & 67 & 33. & 0 & 0 & 0 & \\
\hline
\end{tabular}

Trained with JAFFE + then video surveillance, tested on video surveillance

\begin{tabular}{|c|c|c|c|c|c|c|c|}
\hline \multirow{4}{*}{ 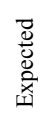 } & $\%$ & $\mathrm{NE}$ & HA & $\mathrm{AG}$ & Prec. & Recall & F1 \\
\hline & $\mathrm{NE}$ & 40 & 60 & 0 & 0.35 & $\begin{array}{l}0.4 \\
\end{array}$ & \multirow{3}{*}{0.19} \\
\hline & HA & 66.6 & 20 & 13.4 & 0.21 & 0.2 & \\
\hline & AG & 33 & 67 & 0 & 0 & 0 & \\
\hline
\end{tabular}

Trained with AffectNet then video surveillance, tested on video surveillance

\begin{tabular}{|c|c|c|c|c|c|c|c|}
\hline \multirow{4}{*}{ 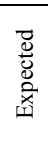 } & $\%$ & $\mathrm{NE}$ & HA & $\mathrm{AG}$ & Prec. & Recall & F1 \\
\hline & $\mathrm{NE}$ & 60 & 13.3 & 26.7 & 0.6 & 0.6 & \multirow{3}{*}{0.37} \\
\hline & HA & 26.7 & 40.0 & 33.3 & 0.66 & 0.4 & \\
\hline & $\mathrm{AG}$ & 67 & 33.0 & 0 & 0.4 & 0.3 & \\
\hline
\end{tabular}

Table 7. Confusion matrix (\%) of the best model (last-75 model) when tested on video surveillance dataset (Exp. 3)

\section{Conclusion and Future Work}

This paper presents the first work in proposing a deep residual learning based approach for measuring customer experience using video surveillance. The model is inspired by ResNet-50 to classify facial expressions into three output classes, which correspond to positive, negative and neutral in order to measure customer experience at an airport context. The paper specifically addresses the main challenge when dealing with limited data availability of video surveillance dataset labelled with facial expression. This paper proposes to adopt knowledge transfer approach, which reuses an existing model trained for image classifications tasks, and retrain it with existing datasets to be suitable for performing facial expression classification tasks on video surveillance data.

The experiments evaluate the proposed model on four different datasets and the results confirm that adopting a residual network as the base model, which retains ImageNet's weights, is a good start to build a reliable model. Adjusting the number of last layers trained can make the model more suitable to the target problem domain. When the video surveillance dataset is still very limited, our experiments show that the models trained using existing similar datasets can work for the video surveillance domain quite effectively.

The experiments also show that the proposed knowledge transfer approach is beneficial as the proposed models do not require a large number of epochs to work for the video surveillance data. However, the performance can still be improved by increasing the number of training images of spontaneous facial expression.

For future works, we will continue expanding the video surveillance dataset and improve the uniformity and quality of the images for improving the training results, which means that we will carefully consider facial pose variations and image resolution, which are some of the key 
issues in dealing with video surveillance as shown by our experiments.

\section{References}

[1] A. K. Smith and R. N. Bolton, "The effect of customers' emotional responses to service failures on their recovery effort evaluations and satisfaction judgments," Journal of the academy of marketing science, vol. 30, no. 1, pp. 5-23, 2002.

[2] O. Harari, "Thank heaven for complainers," Management Review, vol. 81, no. 1, p. 59, 1992.

[3] McKinsey\&Company, "Customer Experience: Creating Value through Transfroming Customer Journeys,"

[4] A. Mollahosseini, B. Hasani, and M. H. Mahoor, "Affectnet: A database for facial expression, valence, and arousal computing in the wild," arXiv preprint arXiv:1708.03985, 2017.

[5] A. Mollahosseini, D. Chan, and M. H. Mahoor, "Going deeper in facial expression recognition using deep neural networks," in Applications of Computer Vision (WACV), 2016 IEEE Winter Conference on, 2016, pp. 1-10: IEEE.

[6] K. Grm, V. Štruc, A. Artiges, M. Caron, and H. K. Ekenel, "Strengths and Weaknesses of Deep Learning Models for Face Recognition against Image Degradations," IET Biometrics, vol. 7, no. 1, pp. 81-89, 2017.

[7] Y. Kim, B. Yoo, Y. Kwak, C. Choi, and J. Kim, "Deep generative-contrastive networks for facial expression recognition," arXiv preprint arXiv:1703.07140, 2017.

[8] K. He, X. Zhang, S. Ren, and J. Sun, "Deep residual learning for image recognition," in Proceedings of the IEEE conference on computer vision and pattern recognition, 2016, pp. 770-778.

[9] T. Kanade, J. F. Cohn, and Y. Tian, "Comprehensive database for facial expression analysis," in Automatic Face and Gesture Recognition, 2000. Proceedings. Fourth IEEE International Conference on, 2000, pp. 46-53: IEEE.

[10] P. Lucey, J. F. Cohn, T. Kanade, J. Saragih, Z. Ambadar, and I. Matthews, "The extended cohn-kanade dataset (ck+): A complete dataset for action unit and emotion-specified expression," in Computer Vision and Pattern Recognition Workshops (CVPRW), 2010 IEEE Computer Society Conference on, 2010, pp. 94-101: IEEE.

[11] M. Lyons, S. Akamatsu, M. Kamachi, and J. Gyoba, "Coding facial expressions with gabor wavelets," in Automatic Face and Gesture Recognition, 1998. Proceedings. Third IEEE International Conference on, 1998, pp. 200-205: IEEE.

[12] X. Zhao et al., "Peak-piloted deep network for facial expression recognition," in European conference on computer vision, 2016, pp. 425-442: Springer.

[13] İ. Çuğu, E. Şener, and E. Akbaş, "MicroExpNet: An Extremely Small and Fast Model For Expression Recognition From Frontal Face Images," arXiv preprint arXiv:1711.07011, 2017.

[14] Z. Yu and C. Zhang, "Image based static facial expression recognition with multiple deep network learning," in
Proceedings of the 2015 ACM on International Conference on Multimodal Interaction, 2015, pp. 435-442: ACM.

[15] P. Liu, S. Han, Z. Meng, and Y. Tong, "Facial expression recognition via a boosted deep belief network," in Proceedings of the IEEE Conference on Computer Vision and Pattern Recognition, 2014, pp. 1805-1812.

[16] L. Surace, M. Patacchiola, E. Battini Sönmez, W. Spataro, and A. Cangelosi, "Emotion recognition in the wild using deep neural networks and Bayesian classifiers," in Proceedings of the 19th ACM International Conference on Multimodal Interaction, 2017, pp. 593-597: ACM.

[17] C. Fabian Benitez-Quiroz, R. Srinivasan, and A. M. Martinez, "Emotionet: An accurate, real-time algorithm for the automatic annotation of a million facial expressions in the wild," in Proceedings of the IEEE Conference on Computer Vision and Pattern Recognition, 2016, pp. 55625570.

[18] M. Parchami, S. Bashbaghi, and E. Granger, "Cnns with cross-correlation matching for face recognition in video surveillance using a single training sample per person," in Advanced Video and Signal Based Surveillance (AVSS), 2017 14th IEEE International Conference on, 2017, pp. 16: IEEE.

[19] C. Herrmann, D. Willersinn, and J. Beyerer, "Lowresolution convolutional neural networks for video face recognition," in Advanced Video and Signal Based Surveillance (AVSS), 2016 13th IEEE International Conference on, 2016, pp. 221-227: IEEE.

[20] Y. Wang, T. Bao, C. Ding, and M. Zhu, "Face recognition in real-world surveillance videos with deep learning method," in Image, Vision and Computing (ICIVC), 2017 2nd International Conference on, 2017, pp. 239-243: IEEE.

[21] Y. Chen, S. Duffner, A. Stoian, J.-Y. Dufour, and A. Baskurt, "Triplet $\mathrm{CNN}$ and pedestrian attribute recognition for improved person re-identification," in Advanced Video and Signal Based Surveillance (AVSS), 2017 14th IEEE International Conference on, 2017, pp. 1-6: IEEE.

[22] H.-W. Ng, V. D. Nguyen, V. Vonikakis, and S. Winkler, "Deep learning for emotion recognition on small datasets using transfer learning," in Proceedings of the 2015 ACM on international conference on multimodal interaction, 2015, pp. 443-449: ACM.

[23] K. Simonyan and A. Zisserman, "Very deep convolutional networks for large-scale image recognition," arXiv preprint arXiv:1409.1556, 2014.

[24] P. Ekman and E. L. Rosenberg, What the face reveals: Basic and applied studies of spontaneous expression using the Facial Action Coding System (FACS). Oxford University Press, USA, 1997.

[25] Microsoft Azure Face API. Available: https://azure.microsoft.com/en-us/services/cognitiveservices/emotion/

[26] Face ++ . Available: https://www.faceplusplus.com/emotion-recognition/

[27] Emovu. Available: http://emovu.com/docs

[28]P. Viola and M. J. Jones, "Robust real-time face detection," International journal of computer vision, vol. 57, no. 2, pp. 137-154, 2004. 